\title{
UNIVERSALITY LIMITS INVOLVING ORTHOGONAL POLYNOMIALS ON THE UNIT CIRCLE
}

\author{
ELI LEVIN ${ }^{1}$ AND DORON S. LUBINSKY ${ }^{2}$
}

\begin{abstract}
We establish universality limits for measures on the unit circle. Assume that $\mu$ is a regular measure on the unit circle in the sense of Stahl and Totik, and is absolutely continuous in an open arc containing some point $z=e^{i \theta}$. Assume, moreover, that $\mu^{\prime}$ is positive and continuous at $z$. Then universality for $\mu$ holds at $z$, in the sense that the normalized reproducing kernel $\tilde{K}_{n}(z, t)$ satisfies
\end{abstract}

$$
\begin{aligned}
& \lim _{n \rightarrow \infty} \frac{1}{n} \tilde{K}_{n}\left(\exp \left(i\left(\theta+\frac{2 \pi a}{n}\right)\right), \exp \left(i\left(\theta+\frac{2 \pi b}{n}\right)\right)\right) \\
= & e^{i \pi(a-b)} \frac{\sin \pi(b-a)}{\pi(b-a)}
\end{aligned}
$$

uniformly for $a, b$ in compact subsets of the real line.

\section{Introduction and Results ${ }^{1}$}

Let $\mu$ be a finite positive Borel measure on $[-\pi, \pi)$ with infinitely many points in its support. Then we may define orthonormal polynomials

$$
\phi_{n}(z)=\kappa_{n} z^{n}+\ldots, \kappa_{n}>0,
$$

$n=0,1,2, \ldots$ satisfying the orthonormality conditions

$$
\frac{1}{2 \pi} \int_{-\pi}^{\pi} \phi_{n}(z) \overline{\phi_{m}(z)} d \mu(\theta)=\delta_{m n},
$$

where $z=e^{i \theta}$. We shall usually assume that $\mu$ is regular in the sense of Stahl and Totik [25], so that

$$
\lim _{n \rightarrow \infty} \kappa_{n}^{1 / n}=1
$$

This is true if for example $\mu^{\prime}>0$ a.e. in $[-\pi, \pi)$, but there are pure jump and pure singularly continuous measures that are regular.

The $n$th reproducing kernel for $\mu$ is

$$
K_{n}(z, u)=\sum_{j=0}^{n-1} \phi_{j}(z) \overline{\phi_{j}(u)} .
$$

Date: September 20, 2007.

${ }^{1}$ Research supported by NSF grant DMS0400446 and US-Israel BSF grant 2004353 
We also use the normalized reproducing kernel

$$
\tilde{K}_{n}\left(e^{i \theta}, e^{i s}\right)=w(\theta)^{1 / 2} w(s)^{1 / 2} K_{n}\left(e^{i \theta}, e^{i s}\right),
$$

where

$$
w(\theta)=\mu^{\prime}(\theta)
$$

Our main result is:

\section{Theorem 1.1}

Let $\mu$ be a finite positive Borel measure on $[-\pi, \pi)$ that is regular. Let $J \subset(-\pi, \pi)$ be compact, and such that $\mu$ is absolutely continuous in an open set containing J. Assume moreover, that $w=\mu^{\prime}$ is positive and continuous at each point of $J$. Then uniformly for $\theta \in J$ and $a, b$ in compact subsets of the real line, we have

$$
\begin{aligned}
& \lim _{n \rightarrow \infty} \frac{\tilde{K}_{n}\left(\exp \left(i\left(\theta+\frac{2 \pi a}{\tilde{K}_{n}\left(e^{i \theta}, e^{i \theta}\right)}\right)\right), \exp \left(i\left(\theta+\frac{2 \pi b}{\tilde{K}_{n}\left(e^{i \theta}, e^{i \theta}\right)}\right)\right)\right)}{\tilde{K}_{n}\left(e^{i \theta}, e^{i \theta}\right)} \\
= & e^{i \pi(a-b)} \frac{\sin \pi(b-a)}{\pi(b-a)} .
\end{aligned}
$$

If $J$ consists of just a single point $\theta$, then the hypothesis is that $\mu$ is absolutely continuous in some neighborhood $(\theta-\varepsilon, \theta+\varepsilon)$ of $\theta$, while $w(\theta)>$ 0 and $w$ is continuous at $\theta$. This alone is sufficient for universality at $\theta$. An alternative way to formulate the limit is that

$$
\lim _{n \rightarrow \infty} \frac{1}{n} \tilde{K}_{n}\left(e^{i\left(\theta+\frac{2 \pi a}{n}\right)}, e^{i\left(\theta+\frac{2 \pi b}{n}\right)}\right)=e^{i \pi(a-b)} \frac{\sin \pi(a-b)}{\pi(a-b)} .
$$

A related, and perhaps more elegant, formulation is

$$
\lim _{n \rightarrow \infty} \frac{K_{n}\left(z\left(1+\frac{i 2 \pi a}{n}\right), z\left(1+\frac{i 2 \pi \bar{b}}{n}\right)\right)}{K_{n}(z, z)}=e^{i \pi(a-b)} \frac{\sin \pi(a-b)}{\pi(a-b)},
$$

uniformly for $a, b$ in compact subsets of the complex plane and $z=e^{i \theta}, \theta \in J$. Note that since $w$ is defined only on $[-\pi, \pi)$, we cannot replace $K_{n}$ by $\tilde{K}_{n}$ in (1.5) even if we restrict $a, b$ to be real. This alternative is proved in Section 6.

Together with Hurwitz's theorem on zeros of sequences of analytic functions, this limit shows that there are zeros of $K_{n}$ spaced roughly $\frac{2 \pi i}{n} z$ apart, in neighborhoods of $z=e^{i \theta}, \theta \in J$ - compare [11]. There is an extensive recent literature on spacing of zeros of orthogonal polynomials on the unit circle [9], [23], [24], and also of paraorthogonal polynomials [28]. The latter are essentially the reproducing kernel $K_{n}(z, z)$ multiplied by a linear factor.

Our universality result on the unit circle is the analogue of a result for $[-1,1]$ proved in [13], and for other situations on the real line in [10], [14]. 
There the increment is $\frac{a}{\tilde{K}_{n}(x, x)}$, rather than $\frac{2 \pi a}{\tilde{K}_{n}\left(e^{i \theta}, e^{i \theta}\right)}$. We need the extra $2 \pi$ in the formulation above, because of the factor $2 \pi$ in the orthonormality condition (1.1).

Like the result in [13], Theorem 1.1 is proved by reducing the measure $\mu$ to normalized Lebesgue measure (or the Legendre weight 1) on the circle. We shall let $\mu^{L}$ denote this measure, that is

$$
d \mu^{L}(\theta)=d \theta \text { on }[-\pi, \pi) .
$$

Note that when forming the orthogonal polynomials, the factor $2 \pi$ in (1.1) ensures that $\mu^{L}$ reduces to normalized Lebesgue measure on the unit circle.

Universality limits are a major topic within random matrix theory, typically for measures on subsets of the real line - see for example, [1], [4], [6], [18]. They have been explored less for general measures on the unit circle. However, for specific measures, such as that occurring in Dyson's circular ensemble, there has been a thorough investigation [18, Chapters 9 to 11].

There are a host of recent results for orthogonal polynomials on the unit circle that have some connection to the results of this paper. In particular, the powerful asymptotics developed using Rieman-Hilbert methods and the $\bar{\partial}$ method [5], [15], [17], yield universality on the unit circle respectively for analytic and Lipschitz continuous weights - not just fixed weights, but varying weights as well.

\section{Corollary 1.2}

Assume the hypotheses of Theorem 1.1. Let $r, s$ be non-negative integers and

$$
K_{n}^{(r, s)}(z, z)=\sum_{k=0}^{n-1} \phi_{k}^{(r)}(z) \overline{\phi_{k}^{(s)}(z)} .
$$

Then uniformly for $\theta \in J, z=e^{i \theta}$,

$$
\lim _{n \rightarrow \infty} \frac{z^{r-s}}{n^{r+s}} \frac{K_{n}^{(r, s)}(z, z)}{K_{n}(z, z)}=\frac{1}{r+s+1} .
$$

In the sequel $C, C_{1}, C_{2}, \ldots$ denote constants independent of $n, z, u, \theta, s, t$. The same symbol does not necessarily denote the same constant in different occurences. We shall write $C=C(\alpha)$ or $C \neq C(\alpha)$ to respectively denote dependence on, or independence of, the parameter $\alpha$. Given measures $\mu^{*}$, $\mu^{\#}$, we use $K_{n}^{*}, K_{n}^{\#}$ and $\phi_{n}^{*}, \phi_{n}^{\#}$ to denote respectively their reproducing kernels and orthonormal polynomials. Similarly superscripts $*$, \# are used to distinguish other quantities associated with them. The superscript $L$ denotes quantities associated with the Legendre weight 1 on $[-\pi, \pi)$. For $s \in \mathbb{R}$ and $\delta>0$, we set

$$
I(s, \delta)=[s-\delta, s+\delta]
$$


The distance from a point $s$ to a set of real numbers $J$ is denoted $\operatorname{dist}(s, J)$. For such a set $J$, we set

$$
J(\delta)=\{s \in \mathbb{R}: \operatorname{dist}(s, J) \leq \delta\} .
$$

$[x]$ denotes the greatest integer $\leq x$. We denote the $n$th Christoffel function for the measure $\mu$ by

$$
\Omega_{n}\left(e^{i \theta}\right)=1 / K_{n}\left(e^{i \theta}, e^{i \theta}\right)=\min _{\operatorname{deg}(P) \leq n-1}\left(\frac{1}{2 \pi} \int_{-\pi}^{\pi}\left|P\left(e^{i t}\right)\right|^{2} d \mu(t)\right) /\left|P\left(e^{i \theta}\right)\right|^{2} .
$$

Note that Máté, Nevai and Totik [16, p. 2, p. 11] used instead lower case $\omega_{n}$, to distinguish it from the Christoffel function $\lambda_{n}$ on the real line. We use the upper case to avoid confusion with $w=\mu^{\prime}$.

As in [13], the main idea in this paper is a localization principle. We use it repeatedly in various forms, but the following basic inequality is typical. Suppose that $\mu, \mu^{*}$ are measures with $\mu \leq \mu^{*}$ in $[-\pi, \pi)$. Then for $|z|,|t|=1$,

$$
\begin{aligned}
& \left|K_{n}(z, t)-K_{n}^{*}(z, t)\right| / K_{n}(z, z) \\
\leq & \left(\frac{K_{n}(t, t)}{K_{n}(z, z)}\right)^{1 / 2}\left[1-\frac{K_{n}^{*}(z, z)}{K_{n}(z, z)}\right]^{1 / 2} \\
= & \left(\frac{\Omega_{n}(z)}{\Omega_{n}(t)}\right)^{1 / 2}\left[1-\frac{\Omega_{n}(z)}{\Omega_{n}^{*}(z)}\right]^{1 / 2} .
\end{aligned}
$$

The paper is organised as follows. In Section 2, we prove some of the results for the Legendre weight. In Section 3, we present some asymptotics for Christoffel functions. In Section 4, we prove our localization principle, including the above inequality. In Section 5, we approximate locally the measure $\mu$ in Theorem 1.1 by a scaled Legendre weight and then prove Theorem 1.1. In Section 6, we prove Corollary 1.2 and the limit (1.5).

\section{The Legendre Weight}

For the Legendre weight (or normalized Lebesgue measure) $d \mu^{L}=d \theta$ on the unit circle, we have

$$
\phi_{n}^{L}(z)=z^{n}, n \geq 0 .
$$

The reproducing kernel is

$$
\begin{aligned}
K_{n}^{L}(z, u) & =\sum_{j=0}^{n-1}(z \bar{u})^{j} \\
& =\left\{\begin{array}{ll}
\frac{1-(z \bar{u})^{n}}{1-z \bar{u}}, & z \neq 1 / \bar{u} \\
n, & z=1 / \bar{u}
\end{array} .\right.
\end{aligned}
$$

Moreover,

$$
\left|K_{n}^{L}(z, u)\right| \leq n \text { for }|z|,|u|=1
$$


and

$$
\frac{1}{2 \pi} \int_{-\pi}^{\pi}\left|K_{n}^{L}\left(z, e^{i t}\right)\right|^{2} d t=n,|z|=1 .
$$

Thus

$$
\Omega_{n}^{L}(z)=\frac{1}{n},|z|=1
$$

Theorem 2.1

(a) Uniformly for $\theta \in[-\pi, \pi)$, and $a, b$ in compact subsets of $\mathbb{C}$,

$$
\begin{aligned}
& \frac{1}{n} K_{n}^{L}\left(e^{i\left(\theta+\frac{2 \pi a}{n}\right)}, e^{i\left(\theta+\frac{2 \pi \bar{b}}{n}\right)}\right) \\
= & e^{i \pi(a-b)} \frac{\sin \pi(a-b)}{\pi(a-b)}+o(1) \\
= & \sum_{r, s=0}^{\infty} \frac{(i 2 \pi a)^{r}(-i 2 \pi b)^{s}}{r ! s !} \frac{1}{r+s+1}+o(1) .
\end{aligned}
$$

(b) The same asymptotic holds for $\frac{1}{n} K_{n}^{L}\left(e^{i \theta}\left(1+\frac{2 \pi i a}{n}\right), e^{i \theta}\left(1+\frac{2 \pi i \bar{b}}{n}\right)\right)$.

Proof

(a) From (2.2),

$$
\begin{aligned}
& \frac{1}{n} K_{n}^{L}\left(e^{i\left(\theta+\frac{a}{n}\right)}, e^{i\left(\theta+\frac{\bar{b}}{n}\right)}\right) \\
= & \frac{1-e^{i(a-b)}}{n\left(1-e^{i(a-b) / n}\right)} \\
= & e^{i\left(\frac{a-b}{2}\right)\left(1-\frac{1}{n}\right)} \frac{\sin \left(\frac{a-b}{2}\right)}{n \sin \left(\frac{a-b}{2 n}\right)}
\end{aligned}
$$


Replacing $a$ by $2 \pi a$ and $b$ by $2 \pi b$, and letting $n \rightarrow \infty$, gives (2.6). The expansion (2.7) follows by observing that

$$
\begin{aligned}
& \frac{1-e^{i(a-b)}}{n\left(1-e^{i(a-b) / n}\right)}+o(1) \\
= & \frac{1-e^{i(a-b)}}{-i(a-b)} \\
= & \sum_{j=0}^{\infty} \frac{(i(a-b))^{j}}{(j+1) !} \\
= & \sum_{j=0}^{\infty} \sum_{k=0}^{j}\left(\begin{array}{l}
j \\
k
\end{array}\right) a^{k}(-b)^{j-k} \frac{i^{j}}{(j+1) !} \\
= & \sum_{k=0}^{\infty} \frac{(i a)^{k}}{k !} \sum_{j=k}^{\infty} \frac{(-i b)^{j-k}}{(j-k) !} \frac{1}{j+1} \\
= & \sum_{k=0}^{\infty} \frac{(i a)^{k}}{k !} \sum_{\ell=0}^{\infty} \frac{(-i b)^{\ell}}{\ell !} \frac{1}{\ell+k+1} .
\end{aligned}
$$

Now replace $a$ by $2 \pi a$ and $b$ by $2 \pi b$.

(b) First observe that uniformly in $\theta$, and for $a$ in a compact subset of the plane,

$$
e^{i\left(\theta+\frac{2 \pi a}{n}\right)}=e^{i \theta}\left(1+\frac{i 2 \pi a}{n}\right)+O\left(\frac{1}{n^{2}}\right)
$$

with a similar relation for $e^{i\left(\theta+\frac{2 \pi \bar{b}}{n}\right)}$. Next, observe that

$$
\frac{\partial}{\partial z} K_{n}^{L}(z, u)=\sum_{j=1}^{n-1} j z^{j-1}(\bar{u})^{j}=O\left(n^{2}\right),
$$

for $|u|,|z| \leq 1+\frac{C}{n}$. A similar relation holds for $\frac{\partial}{\partial u}\left[K_{n}^{L}(z, \bar{u})\right]$. Note that $K_{n}^{L}(z, \bar{u})$ is analytic in each of the variables $z$ and $u$. Then an integration in each variable shows that

$\frac{1}{n} K_{n}^{L}\left(e^{i \theta}\left(1+\frac{2 \pi i a}{n}\right), e^{i \theta}\left(1+\frac{2 \pi i \bar{b}}{n}\right)\right)=\frac{1}{n} K_{n}^{L}\left(e^{i\left(\theta+\frac{2 \pi a}{n}\right)}, e^{i\left(\theta+\frac{2 \pi \bar{b}}{n}\right)}\right)+o(1)$, uniformly for $a, b$ in compact subsets of the plane and $\theta \in[-\pi, \pi)$.

In the following result, as in (1.7), we use the notation

$$
\left(K_{n}^{L}\right)^{(r, s)}(z, z)=\sum_{k=0}^{n-1} \phi_{k}^{L(r)}(z) \overline{\phi_{k}^{L(s)}(z)} .
$$

\section{Theorem 2.2}


Let $r, s$ be non-negative integers. Then uniformly for $\theta \in[-\pi, \pi), z=e^{i \theta}$,

$$
\lim _{n \rightarrow \infty} \frac{z^{r-s}}{n^{r+s+1}}\left(K_{n}^{L}\right)^{(r, s)}(z, z)=\frac{1}{r+s+1} .
$$

\section{Proof}

Taylor series expansion shows that

$$
\begin{aligned}
& \frac{1}{n} K_{n}^{L}\left(z\left(1+\frac{i 2 \pi a}{n}\right), z\left(1+\frac{i 2 \pi \bar{b}}{n}\right)\right) \\
= & \frac{1}{n} \sum_{k=0}^{n-1} \phi_{k}^{L}\left(z+\frac{i 2 \pi a z}{n}\right) \overline{\phi_{k}^{L}\left(z+\frac{i 2 \pi \bar{b} z}{n}\right)} \\
= & \frac{1}{n} \sum_{k=0}^{n-1}\left(\sum_{r=0}^{\infty} \frac{\phi_{k}^{L(r)}(z)}{r !}\left(\frac{i 2 \pi a z}{n}\right)^{r}\right) \overline{\left(\sum_{s=0}^{\infty} \frac{\phi_{k}^{L(s)}(z)}{s !}\left(\frac{i 2 \pi \bar{b} z}{n}\right)^{s}\right)} \\
= & \sum_{r, s=0}^{\infty} \frac{(i 2 \pi a)^{r}(-i 2 \pi b)^{s}}{r ! s !} \frac{z^{r-s}\left(K_{n}^{L}\right)^{(r, s)}(z, z)}{n^{r+s+1}} .
\end{aligned}
$$

The interchanges are justified, since the series all terminate. Recall that when sequences of analytic functions converge uniformly, their Taylor series coefficients converge to those of the limit function. Then comparing with (2.7) of Theorem 2.1, and using (b) there, gives the result.

\section{Christoffel functions}

We use $\Omega_{n}^{L}$ to denote the $n$th Christoffel function for the Legendre weight (or normalized Lebesgue measure) $\frac{d \theta}{2 \pi}$ on $[-\pi, \pi)$. The methods used to prove the following result are well known [16], [19], [20], [27], but we need the increment $a / n$ which is absent from results in the literature. An interesting new method to prove weak convergence of the Christoffel functions, has been provided in [2].

\section{Theorem 3.1}

Let $\mu$ be a regular measure on $[-\pi, \pi)$. Assume that $\mu$ is absolutely continuous in an open set containing a compact set $J$, and at each point of $J, w=\mu^{\prime}$ is positive and continuous. Let $A>0$. Then uniformly for $a \in[-A, A]$, and $z=e^{i \theta} \in J$,

$$
\lim _{n \rightarrow \infty} n \Omega_{n}\left(\exp \left(i\left(\theta+\frac{a}{n}\right)\right)\right)=w(\theta) .
$$

Moreover, uniformly for $n \geq n_{0}(A), \theta \in J$, and $a \in[-A, A]$,

$$
\Omega_{n}\left(\exp \left(i\left(\theta+\frac{a}{n}\right)\right)\right) \sim \frac{1}{n} .
$$

\section{Remarks}

(a) The notation $\sim$ means that the ratio of the two quantities is bounded 
above and below by positive constants independent of $n, \theta$ and $a$.

(b) We emphasize that we are assuming that $w$ is continuous in $J$ when regarded as a function defined on $[-\pi, \pi)$.

\section{Proof}

Let $\varepsilon>0$ and choose $\delta>0$ such that $\mu$ is absolutely continuous in $J(\delta) \subset$ $[-\pi, \pi)$, and such that

$$
(1+\varepsilon)^{-1} \leq \frac{w(s)}{w(t)} \leq 1+\varepsilon, s, t \in J(\delta) \text { with }|s-t| \leq \delta .
$$

(This is possible because of compactness of $J$ and continuity and positivity of $w$ at every point of $J)$. Let us fix $s_{0} \in J$, so that $s_{0} \in(-\pi, \pi)$ and recall that $I\left(s_{0}, \delta\right)=\left[s_{0}-\delta, s_{0}+\delta\right]$. Define a measure $\mu^{*}$ with

$$
\mu^{*}=\mu \text { in }[-\pi, \pi) \backslash I\left(s_{0}, \delta\right)
$$

and in $I\left(s_{0}, \delta\right)$, let $\mu^{*}$ be absolutely continuous, with $w^{*}=\left(\mu^{*}\right)^{\prime}$ satisfying

$$
w^{*}=w\left(s_{0}\right)(1+\varepsilon) \text { in } I\left(s_{0}, \delta\right) .
$$

Because of (3.3), $d \mu \leq d \mu^{*}$ in $[-\pi, \pi)$. Then, if $\Omega_{n}^{*}$ is the $n$th Christoffel function for $\mu^{*}$, the extremal property (1.9) gives everywhere,

$$
\Omega_{n} \leq \Omega_{n}^{*} \text {. }
$$

We now find an upper bound for $\Omega_{n}^{*}(s)$ for $s \in I\left(s_{0}, \delta / 2\right)$. Note that for all real $s, t$,

$$
\left|\frac{1}{2}\left(1+e^{i(t-s)}\right)\right|^{2}=\cos ^{2}\left(\frac{t-s}{2}\right) \leq 1,
$$

and there exists $r \in(0,1)$ depending only on $\delta$ such that

$$
0 \leq\left|\frac{1}{2}\left(1+e^{i(t-s)}\right)\right| \leq r \text { for } s \in I\left(s_{0}, \delta / 2\right) \text { and } t \in[-\pi, \pi) \backslash I\left(s_{0}, \delta\right) .
$$

Fix $0<\eta<\frac{1}{2}$ and let $m=m(n)=n-[\eta n]$. Fix $s \in I\left(s_{0}, \delta / 2\right)$ and let

$$
S_{n}\left(e^{i t}\right)=\frac{1}{m} K_{m}^{L}\left(e^{i s}, e^{i t}\right)\left(\frac{1}{2}\left(1+e^{i(t-s)}\right)\right)^{[\eta n]},
$$

a polynomial of degree $\leq m-1+[\eta n]=n-1$ with $S_{n}\left(e^{i s}\right)=1$. Then using (2.3), (3.4), and (3.7),

$$
\begin{aligned}
\Omega_{n}\left(e^{i s}\right) & \leq \Omega_{n}^{*}\left(e^{i s}\right) \leq \frac{1}{2 \pi} \int_{-\pi}^{\pi}\left|S_{n}\left(e^{i t}\right)\right|^{2} d \mu^{*}(t) \\
& \leq \frac{w\left(s_{0}\right)(1+\varepsilon)}{2 \pi m^{2}} \int_{I\left(s_{0}, \delta\right)}\left|K_{m}^{L}\left(e^{i s}, e^{i t}\right)\right|^{2} d t+r^{2[\eta n]} \int_{[-\pi, \pi) \backslash I\left(s_{0}, \delta\right)} d \mu^{*} \\
& \leq \frac{w\left(s_{0}\right)(1+\varepsilon)}{m}(1+o(1)) \\
& \leq \frac{w\left(s_{0}\right)}{n} \frac{1+\varepsilon}{1-\eta}(1+o(1)) .
\end{aligned}
$$


Here we also used (2.4) and our choice $m=n-[\eta n]$ of $m$. Thus for some $n_{0}=n_{0}\left(s_{0}\right)$ and for $n \geq n_{0}$,

$$
\sup _{s \in I\left(s_{0}, \delta / 2\right)} n \Omega_{n}\left(e^{i s}\right) \leq w\left(s_{0}\right) \frac{1+2 \varepsilon}{1-\eta} .
$$

Recalling (3.3), and covering $J$ with finitely many such intervals $I\left(s_{0}, \delta / 2\right)$, we obtain for some maximal threshhold $n_{1}$, that for $n \geq n_{1}=n_{1}(\varepsilon, \delta, J)$,

$$
\sup _{s \in J(\delta / 2)} n \Omega_{n}\left(e^{i s}\right) / w(s) \leq \frac{(1+2 \varepsilon)^{2}}{1-\eta} .
$$

Now let $A>0$ and $|a| \leq A$. There exists $n_{2}=n_{2}(A)$ such that for $n \geq n_{2}$ and all $|a| \leq A$ and all $s \in J$, we have $s+\frac{a}{n} \in J(\delta / 2)$. We deduce, using (3.3) again, that

$$
\limsup _{n \rightarrow \infty}\left(\sup _{a \in[-A, A], s \in J} n \Omega_{n}\left(e^{i\left(s+\frac{a}{n}\right)}\right) / w(s)\right) \leq \frac{(1+2 \varepsilon)^{3}}{1-\eta} .
$$

As the left-hand side is independent of the parameters $\varepsilon, \eta$, we deduce that

$$
\limsup _{n \rightarrow \infty}\left(\sup _{a \in[-A, A], s \in J} n \Omega_{n}\left(e^{i\left(s+\frac{a}{n}\right)}\right) / w(s)\right) \leq 1 .
$$

In a somewhat more technical way, we can establish the converse bound

$$
\limsup _{n \rightarrow \infty}\left(\sup _{a \in[-A, A], s \in J} \frac{w(s)}{n \Omega_{n}\left(e^{i\left(s+\frac{a}{n}\right)}\right)}\right) \leq 1 .
$$

Let $\eta \in\left(0, \frac{1}{2}\right)$ and $r$ be as at (3.7). Choose $\sigma>1$ so close to 1 that

$$
\sigma^{1-\eta}<r^{-\eta / 4} \text {. }
$$

Let $m=m(n)=n-[\eta n]$. Because $\mu$ is a regular measure, we have $[25, \mathrm{p}$ $66]$

$$
\sup _{\operatorname{deg}(P) \leq \ell}\left(\frac{\|P\|_{L_{\infty}(|z|=1)}^{2}}{\frac{1}{2 \pi} \int_{-\pi}^{\pi}\left|P\left(e^{i t}\right)\right|^{2} d \mu(t)}\right)^{1 / \ell} \leq 1+o(1), \ell \rightarrow \infty .
$$

Hence for $n$ large enough, and all polynomials $P$ of degree $\leq m=n-[\eta n]$,

$$
\|P\|_{L_{\infty}(|z|=1)}^{2} \leq \frac{\left(\sigma^{1-\eta}\right)^{2 n}}{2 \pi} \int_{-\pi}^{\pi}\left|P\left(e^{i t}\right)\right|^{2} d \mu(t) .
$$

Fix $s \in I\left(s_{0}, \delta / 2\right)$ and let

$$
S_{n}\left(e^{i t}\right)=\frac{K_{m}\left(e^{i s}, e^{i t}\right)}{K_{m}\left(e^{i s}, e^{i s}\right)}\left(\frac{1+e^{i(t-s)}}{2}\right)^{[\eta n]} .
$$

Then $S_{n}\left(e^{i s}\right)=1$, so (2.5), followed by (3.3) and (3.7), gives

$$
\frac{1}{n}=\Omega_{n}^{L}\left(e^{i s}\right) \leq \frac{1}{2 \pi} \int_{-\pi}^{\pi}\left|S_{n}\right|^{2}
$$




$$
\begin{aligned}
\leq & \frac{\left[w\left(s_{0}\right)^{-1}(1+\varepsilon)\right]}{2 \pi} \int_{I\left(s_{0}, \delta\right)}\left|\frac{K_{m}\left(e^{i s}, e^{i t}\right)}{K_{m}\left(e^{i s}, e^{i s}\right)}\right|^{2} w(t) d t \\
& +\|\left.\frac{K_{m}\left(e^{i s}, e^{i \cdot}\right)}{K_{m}\left(e^{i s}, e^{i s}\right)}\right|_{L_{\infty}\left([-\pi, \pi) \backslash I\left(s_{0}, \delta\right)\right)} ^{2} \frac{r^{2[\eta n]}}{2 \pi} \int_{[-\pi, \pi) \backslash I\left(s_{0}, \delta\right)} 1 \\
\leq & \frac{\left[w\left(s_{0}\right)^{-1}(1+\varepsilon)\right]}{2 \pi} \int_{-\pi}^{\pi}\left|\frac{K_{m}\left(e^{i s}, e^{i t}\right)}{K_{m}\left(e^{i s}, e^{i s}\right)}\right|^{2} d \mu(t)\left(1+C\left(\sigma^{1-\eta} r^{\eta}\right)^{2 n}\right) \\
= & w\left(s_{0}\right)^{-1}(1+\varepsilon) \Omega_{m}\left(e^{i s}\right)(1+o(1)),
\end{aligned}
$$

recall (3.11) and (3.10). Thus

$$
\frac{1}{\Omega_{m}\left(e^{i s}\right)} \leq n w\left(s_{0}\right)^{-1}(1+\varepsilon)(1+o(1)) .
$$

Then, as above

$$
\begin{aligned}
& \sup _{s \in I\left(s_{0}, \delta / 2\right)} \frac{1}{m \Omega_{m}\left(e^{i s}\right)} \\
\leq & {\left[w\left(s_{0}\right)^{-1}(1+\varepsilon)(1+o(1))\right] \frac{n}{m} } \\
\leq & {\left[w\left(s_{0}\right)^{-1}\left(\frac{1+\varepsilon}{1-\eta}\right)\right]\{1+o(1)\} . }
\end{aligned}
$$

As $n$ runs through all the positive integers, so does $m=n-[\eta n]$. (Indeed, the difference between successive such $m$ is at most 1). Then (3.9) follows using monotonicity of $\Omega_{n}$ in $n$, much as above. Together (3.8) and (3.9) give (3.1). Finally, (3.2) follows from (3.1).

\section{LOCALIZATION}

\section{Theorem 4.1}

Assume that $\mu, \mu^{*}$ are regular measures on $[-\pi, \pi)$ that are absolutely continuous in an open set containing a compact set $J$. Assume that $w=\mu^{\prime}$ is positive and continuous in $J$ and

$$
d \mu=d \mu^{*} \text { in } J
$$

Let $A>0$. Then as $n \rightarrow \infty$,

$$
\sup _{a, b \in[-A, A], s \in J}\left|\left(K_{n}-K_{n}^{*}\right)\left(e^{i\left(s+\frac{a}{n}\right)}, e^{i\left(s+\frac{b}{n}\right)}\right)\right| / n=o(1) .
$$

\section{Proof}

We initially assume that

$$
d \mu \leq d \mu^{*} \text { in }[-\pi, \pi) .
$$


The idea is to estimate the $L_{2}$ norm of $\left(K_{n}-K_{n}^{*}\right)\left(e^{i s}, e^{i t}\right)$ over $[-\pi, \pi)$, and then to use Christoffel function estimates. Now

$$
\begin{aligned}
& \frac{1}{2 \pi} \int_{-\pi}^{\pi}\left|\left(K_{n}-K_{n}^{*}\right)\left(e^{i s}, e^{i t}\right)\right|^{2} d \mu(t) \\
= & \frac{1}{2 \pi} \int_{-\pi}^{\pi}\left|K_{n}^{2}\left(e^{i s}, e^{i t}\right)\right| d \mu(t)-\frac{2}{2 \pi} \operatorname{Re} \int_{-\pi}^{\pi}\left(\overline{K_{n}} K_{n}^{*}\right)\left(e^{i s}, e^{i t}\right) d \mu(t) \\
& +\frac{1}{2 \pi} \int_{-\pi}^{\pi}\left|K_{n}^{* 2}\left(e^{i s}, e^{i t}\right)\right| d \mu(t) \\
= & K_{n}\left(e^{i s}, e^{i s}\right)-2 K_{n}^{*}\left(e^{i s}, e^{i s}\right)+\frac{1}{2 \pi} \int_{-\pi}^{\pi}\left|K_{n}^{*}\left(e^{i s}, e^{i t}\right)\right|^{2} d \mu(t),
\end{aligned}
$$

by the reproducing kernel property. As $d \mu \leq d \mu^{*}$, we also have

$\frac{1}{2 \pi} \int_{-\pi}^{\pi}\left|K_{n}^{*}\left(e^{i s}, e^{i t}\right)\right|^{2} d \mu(t) \leq \frac{1}{2 \pi} \int_{-\pi}^{\pi}\left|K_{n}^{*}\left(e^{i s}, e^{i t}\right)\right|^{2} d \mu^{*}(t)=K_{n}^{*}\left(e^{i s}, e^{i s}\right)$.

So

$$
\begin{aligned}
& \frac{1}{2 \pi} \int_{-\pi}^{\pi}\left|\left(K_{n}-K_{n}^{*}\right)\left(e^{i t}, e^{i s}\right)\right|^{2} d \mu(t) \\
= & \frac{1}{2 \pi} \int_{-\pi}^{\pi}\left|\left(K_{n}-K_{n}^{*}\right)\left(e^{i s}, e^{i t}\right)\right|^{2} d \mu(t) \\
\leq & \left(K_{n}-K_{n}^{*}\right)\left(e^{i s}, e^{i s}\right) .
\end{aligned}
$$

Next for any polynomial $P$ of degree $\leq n-1$, we have the Christoffel function estimate

$$
\left|P\left(e^{i t}\right)\right| \leq K_{n}^{1 / 2}\left(e^{i t}, e^{i t}\right)\left(\frac{1}{2 \pi} \int_{-\pi}^{\pi}\left|P\left(e^{i u}\right)\right|^{2} d \mu(u)\right)^{1 / 2} .
$$

Applying this to $P\left(e^{i t}\right)=\left(K_{n}-K_{n}^{*}\right)\left(e^{i t}, e^{i s}\right)$ and using (4.3) gives, for all $s, t \in[-\pi, \pi)$,

$$
\begin{aligned}
& \left|\left(K_{n}-K_{n}^{*}\right)\left(e^{i t}, e^{i s}\right)\right| \\
\leq & K_{n}^{1 / 2}\left(e^{i t}, e^{i t}\right)\left[\left(K_{n}-K_{n}^{*}\right)\left(e^{i s}, e^{i s}\right)\right]^{1 / 2},
\end{aligned}
$$

so

$$
\begin{aligned}
& \left|\left(K_{n}-K_{n}^{*}\right)\left(e^{i s}, e^{i t}\right)\right| / K_{n}\left(e^{i s}, e^{i s}\right) \\
\leq & \left(\frac{K_{n}\left(e^{i t}, e^{i t}\right)}{K_{n}\left(e^{i s}, e^{i s}\right)}\right)^{1 / 2}\left[1-\frac{K_{n}^{*}\left(e^{i s}, e^{i s}\right)}{K_{n}\left(e^{i s}, e^{i s}\right)}\right]^{1 / 2} .
\end{aligned}
$$

Now we set $s=s_{0}+\frac{a}{n}$ and $t=s_{0}+\frac{b}{n}$, where $a, b \in[-A, A]$ and $s_{0} \in J$. By Theorem 3.1, uniformly for such $s, \frac{K_{n}^{*}\left(e^{i s}, e^{i s}\right)}{K_{n}\left(e^{i s}, e^{i s}\right)}=1+o(1)$, for $\left(\mu^{*}\right)^{\prime}=\mu^{\prime}=w$ in $J$. Moreover, uniformly for $a, b \in[-A, A]$,

$$
K_{n}\left(e^{i\left(s_{0}+\frac{b}{n}\right)}, e^{i\left(s_{0}+\frac{b}{n}\right)}\right) \sim K_{n}\left(e^{i\left(s_{0}+\frac{a}{n}\right)}, e^{i\left(s_{0}+\frac{a}{n}\right)}\right) \sim n,
$$


so

$$
\sup _{a, b \in[-A, A], s_{0} \in J}\left|\left(K_{n}-K_{n}^{*}\right)\left(e^{i\left(s_{0}+\frac{a}{n}\right)}, e^{i\left(s_{0}+\frac{b}{n}\right)}\right)\right| / n=o(1) .
$$

Now we drop the extra hypothesis (4.2). Define a measure $\nu$ by $\nu=\mu=\mu^{*}$ in $J$; and in $[-\pi, \pi) \backslash J$, let

$$
d \nu(t)=\max \left\{\operatorname{dist}(t, J), w(t), w^{*}(t)\right\} d t+d \mu_{s}(t)+d \mu_{s}^{*}(t),
$$

where $w, w^{*}$ and $\mu_{s}, \mu_{s}^{*}$ are respectively the absolutely continuous and singular components of $\mu, \mu^{*}$. Then $d \mu \leq d \nu$ and $d \mu^{*} \leq d \nu$, and $\nu$ is regular as its absolutely continuous component is positive in $[-\pi, \pi)$. Moreover, $\nu$ is absolutely continuous in an open interval containing $J$, and $\nu^{\prime}=w$ in $J$. The case above shows that the reproducing kernels for $\mu$ and $\mu^{*}$ have the same asymptotics as that for $\nu$, in the sense of (4.1), and hence the same asymptotics as each other.

\section{Smoothing}

In this section, we approximate $\mu$ of Theorem 1.1 by a multiple $\mu^{\#}$ of Lebesgue measure, and then prove Theorem 1.1. Recall that $\tilde{K}_{n}$ is the normalized kernel, given by (1.5). Our smoothing result (which may also be viewed as localization) is:

\section{Theorem 5.1}

Let $\mu$ be as in Theorem 1.1. Let $A>0, \varepsilon \in\left(0, \frac{1}{2}\right)$ and choose $\delta>0$ such that (3.3) holds. Let $s_{0} \in J$. Then there exists $n_{0}$ such that for $n \geq n_{0}$,

$$
\sup _{a, b \in[-A, A], s \in I\left(s_{0}, \frac{\delta}{2}\right) \cap J}\left|\left(\tilde{K}_{n}-\tilde{K}_{n}^{L}\right)\left(e^{i\left(s+\frac{a}{n}\right)}, e^{i\left(s+\frac{b}{n}\right)}\right)\right| / n \leq(8 \varepsilon)^{1 / 2} .
$$

\section{Proof}

Fix $s_{0} \in J$ and let $w^{\#}$ be the scaled Legendre weight

$$
w^{\#}=w\left(s_{0}\right) \text { in }[-\pi, \pi) .
$$

Note that

$$
K_{n}^{\#}\left(e^{i s}, e^{i t}\right)=\frac{1}{w\left(s_{0}\right)} K_{n}^{L}\left(e^{i s}, e^{i t}\right) .
$$

(Recall that the superscript $L$ indicates the Legendre weight on $[-\pi, \pi)$ ). Because of our localization result Theorem 4.1 , we may replace $d \mu$ by $w^{*}(t) d t$, where

$$
w^{*}=w \text { in } I\left(s_{0}, \delta\right)
$$

and

$$
w^{*}=w\left(s_{0}\right) \text { in }[-\pi, \pi) \backslash I\left(s_{0}, \delta\right),
$$

without affecting the asymptotics for $K_{n}\left(e^{i\left(s+\frac{a}{n}\right)}, e^{i\left(s+\frac{b}{n}\right)}\right)$ in the interval $I\left(s_{0}, \frac{\delta}{2}\right)$. (Note that $\varepsilon$ and $\delta$ play no role in Theorem 4.1 ). So in the sequel, 
we assume that $w=w\left(s_{0}\right)=w^{\#}$ in $[-\pi, \pi) \backslash I\left(s_{0}, \delta\right)$, while not changing $w$ in $I\left(s_{0}, \delta\right)$. Observe that (3.3) implies that

$$
(1+\varepsilon)^{-1} \leq \frac{w}{w^{\#}} \leq 1+\varepsilon, \text { in }[-\pi, \pi) .
$$

Then, much as in the previous section,

$$
\begin{aligned}
& \frac{1}{2 \pi} \int_{-\pi}^{\pi}\left|\left(K_{n}-K_{n}^{\#}\right)^{2}\left(e^{i s}, e^{i t}\right)\right| w^{\#}(t) d t \\
= & \frac{1}{2 \pi} \int_{-\pi}^{\pi}\left|K_{n}\left(e^{i s}, e^{i t}\right)\right|^{2} w^{\#}(t) d t-\frac{2}{2 \pi} \operatorname{Re} \int_{-\pi}^{\pi}\left(K_{n} \overline{K_{n}^{\#}}\right)\left(e^{i s}, e^{i t}\right) w^{\#}(t) d t \\
& +\frac{1}{2 \pi} \int_{-\pi}^{\pi}\left|K_{n}^{\#}\left(e^{i s}, e^{i t}\right)\right|^{2} w^{\#}(t) d t \\
= & \frac{1}{2 \pi} \int_{-\pi}^{\pi}\left|K_{n}\left(e^{i s}, e^{i t}\right)\right|^{2} w(t) d t+\frac{1}{2 \pi} \int_{I\left(s_{0}, \delta\right)}\left|K_{n}\left(e^{i s}, e^{i t}\right)\right|^{2}\left(w^{\#}-w\right)(t) d t \\
& -2 K_{n}\left(e^{i s}, e^{i s}\right)+K_{n}^{\#}\left(e^{i s}, e^{i s}\right) \\
= & K_{n}^{\#}\left(e^{i s}, e^{i s}\right)-K_{n}\left(e^{i s}, e^{i s}\right)+\frac{1}{2 \pi} \int_{I\left(s_{0}, \delta\right)}\left|K_{n}\left(e^{i s}, e^{i t}\right)\right|^{2}\left(w^{\#}-w\right)(t) d t,
\end{aligned}
$$

recall that $w=w^{\#}$ in $[-\pi, \pi) \backslash I\left(s_{0}, \delta\right)$. By $(5.3)$,

$$
\begin{aligned}
& \frac{1}{2 \pi} \int_{I\left(s_{0}, \delta\right)}\left|K_{n}\left(e^{i s}, e^{i t}\right)\right|^{2}\left(w^{\#}-w\right)(t) d t \\
\leq & \frac{\varepsilon}{2 \pi} \int_{I\left(s_{0}, \delta\right)}\left|K_{n}\left(e^{i s}, e^{i t}\right)\right|^{2} w(t) d t \leq \varepsilon K_{n}\left(e^{i s}, e^{i s}\right) .
\end{aligned}
$$

So

$\frac{1}{2 \pi} \int_{-\pi}^{\pi}\left|\left(K_{n}-K_{n}^{\#}\right)^{2}\left(e^{i s}, e^{i t}\right)\right| w^{\#}(t) d t \leq K_{n}^{\#}\left(e^{i s}, e^{i s}\right)-(1-\varepsilon) K_{n}\left(e^{i s}, e^{i s}\right)$.

Applying an obvious analogue of (4.4) to $P\left(e^{i t}\right)=\left(K_{n}-K_{n}^{\#}\right)\left(e^{i t}, e^{i s}\right)$ and using (5.4) gives for $s, t \in[-\pi, \pi)$,

$$
\begin{aligned}
& \left|\left(K_{n}-K_{n}^{\#}\right)\left(e^{i t}, e^{i s}\right)\right| \\
\leq & K_{n}^{\#}\left(e^{i t}, e^{i t}\right)^{1 / 2}\left[K_{n}^{\#}\left(e^{i s}, e^{i s}\right)-(1-\varepsilon) K_{n}\left(e^{i s}, e^{i s}\right)\right]^{1 / 2}
\end{aligned}
$$

so

$$
\begin{aligned}
& \left|\left(K_{n}-K_{n}^{\#}\right)\left(e^{i s}, e^{i t}\right)\right| / K_{n}^{\#}\left(e^{i s}, e^{i s}\right) \\
\leq & \left(\frac{K_{n}^{\#}\left(e^{i t}, e^{i t}\right)}{K_{n}^{\#}\left(e^{i s}, e^{i s}\right)}\right)^{1 / 2}\left[1-(1-\varepsilon) \frac{K_{n}\left(e^{i s}, e^{i s}\right)}{K_{n}^{\#}\left(e^{i s}, e^{i s}\right)}\right]^{1 / 2} .
\end{aligned}
$$


In view of (5.3), we also have

$$
\frac{K_{n}\left(e^{i s}, e^{i s}\right)}{K_{n}^{\#}\left(e^{i s}, e^{i s}\right)}=\frac{\Omega_{n}^{\#}\left(e^{i s}\right)}{\Omega_{n}\left(e^{i s}\right)} \geq \frac{1}{1+\varepsilon},
$$

so for all $s, t \in[-\pi, \pi)$,

$$
\begin{aligned}
& \left|\left(K_{n}-K_{n}^{\#}\right)\left(e^{i s}, e^{i t}\right)\right| / K_{n}^{\#}\left(e^{i s}, e^{i s}\right) \\
\leq & \left(\frac{K_{n}^{\#}\left(e^{i t}, e^{i t}\right)}{K_{n}^{\#}\left(e^{i s}, e^{i s}\right)}\right)^{1 / 2}\left[1-\frac{1-\varepsilon}{1+\varepsilon}\right]^{1 / 2} \\
\leq & \sqrt{2 \varepsilon}\left(\frac{K_{n}^{\#}\left(e^{i t}, e^{i t}\right)}{K_{n}^{\#}\left(e^{i s}, e^{i s}\right)}\right)^{1 / 2} \\
= & \sqrt{2 \varepsilon}\left(\frac{K_{n}^{L}\left(e^{i t}, e^{i t}\right)}{K_{n}^{L}\left(e^{i s}, e^{i s}\right)}\right)^{1 / 2}=\sqrt{2 \varepsilon} .
\end{aligned}
$$

Here we have used (5.2) and (2.5). Now we set $s=s_{1}+\frac{a}{n}$ and $t=s_{1}+\frac{b}{n}$, where $s_{1} \in I\left(s_{0}, \frac{\delta}{2}\right)$ and $a, b \in[-A, A]$. Then using (5.2) again,

$$
\sup _{a, b \in[-A, A], s_{1} \in I\left(s_{0}, \frac{\delta}{2}\right) \cap J}\left|\left(w\left(s_{0}\right) K_{n}-K_{n}^{L}\right)\left(e^{i\left(s_{1}+\frac{a}{n}\right)}, e^{i\left(s_{1}+\frac{b}{n}\right)}\right)\right| / n \leq \sqrt{2 \varepsilon} .
$$

Next, by (3.3), we have for $n \geq n_{0}, s_{1} \in I\left(s_{0}, \frac{\delta}{2}\right) \cap J$ and $a, b \in[-A, A]$,

$$
(1+\varepsilon)^{-1} \leq \frac{w\left(s_{1}+\frac{a}{n}\right)^{1 / 2} w\left(s_{1}+\frac{b}{n}\right)^{1 / 2}}{w\left(s_{0}\right)} \leq 1+\varepsilon .
$$

Then for such $n, s_{1}, a$ and $b$,

$$
\begin{aligned}
& \left|\left(\tilde{K}_{n}-\tilde{K}_{n}^{L}\right)\left(e^{i\left(s_{1}+\frac{a}{n}\right)}, e^{i\left(s_{1}+\frac{b}{n}\right)}\right)\right| / n \\
= & \left|\left(\frac{w\left(s_{1}+\frac{a}{n}\right)^{1 / 2} w\left(s_{1}+\frac{b}{n}\right)^{1 / 2}}{w\left(s_{0}\right)} w\left(s_{0}\right) K_{n}-K_{n}^{L}\right)\left(e^{i\left(s_{1}+\frac{a}{n}\right)}, e^{i\left(s_{1}+\frac{b}{n}\right)}\right)\right| / n \\
\leq & \left|\frac{w\left(s_{1}+\frac{a}{n}\right)^{1 / 2} w\left(s_{1}+\frac{b}{n}\right)^{1 / 2}}{w\left(s_{0}\right)}-1\right| w\left(s_{0}\right)\left|K_{n}\left(e^{i\left(s_{1}+\frac{a}{n}\right)}, e^{i\left(s_{1}+\frac{b}{n}\right)}\right)\right| / n+\sqrt{2 \varepsilon} \\
\leq & \varepsilon(1+\sqrt{2 \varepsilon})+\sqrt{2 \varepsilon} \leq \sqrt{8 \varepsilon},
\end{aligned}
$$

recall $\varepsilon<\frac{1}{2}$.

\section{Proof of Theorem 1.1}

Let $A, \varepsilon>0$. Choose $\delta>0$ such that (3.3) holds. Now cover $J$ by, say, $M$ intervals $I\left(s_{j}, \frac{\delta}{2}\right), 1 \leq j \leq M$, each of length $\delta$. For each $j$, there exists a threshhold $n_{0}=n_{0}(j)$ for which (5.1) holds for $n \geq n_{0}(j)$ with $I\left(s_{0}, \frac{\delta}{2}\right)$ 
replaced by $I\left(s_{j}, \frac{\delta}{2}\right)$. Let $n_{1}$ denote the largest of these. Then we obtain, for $n \geq n_{1}$,

$$
\sup _{a, b \in[-A, A], s \in J}\left|\left(\tilde{K}_{n}-K_{n}^{L}\right)\left(e^{i\left(s+\frac{a}{n}\right)}, e^{i\left(s+\frac{b}{n}\right)}\right)\right| / n \leq \sqrt{8 \varepsilon} .
$$

It follows that

$$
\lim _{n \rightarrow \infty}\left(\sup _{a, b \in[-A, A], s \in J}\left|\left(\tilde{K}_{n}-K_{n}^{L}\right)\left(e^{i\left(s+\frac{a}{n}\right)}, e^{i\left(s+\frac{b}{n}\right)}\right)\right| / n\right)=0 .
$$

Finally recall Theorem 2.1(a). Thus, replacing $a$ by $2 \pi a$ and $b$ by $2 \pi b$, we obtain,

$$
\frac{1}{n} \tilde{K}_{n}\left(e^{i\left(s+\frac{2 \pi a}{n}\right)}, e^{i\left(s+\frac{2 \pi b}{n}\right)}\right)=e^{i \pi(a-b)} \frac{\sin \pi(a-b)}{\pi(a-b)}+o(1)
$$

uniformly for $s \in J$ and $a, b$ in compact subsets of the real line. This is of course (1.4). Since uniformly for $s \in J$, by Theorem 3.1,

$$
\begin{aligned}
\tilde{K}_{n}\left(e^{i s}, e^{i s}\right)^{-1} & =K_{n}^{L}\left(e^{i s}, e^{i s}\right)^{-1}(1+o(1)) \\
& =n^{-1}(1+o(1)),
\end{aligned}
$$

we then also obtain the conclusion (1.3) of Theorem 1.1.

\section{Proof of Corollary 1.2}

We begin with a bound on the growth of a polynomial close to an arc of the unit circle. For a subset $\mathcal{K}$ of $[-\pi, \pi)$, and $r \geq 1$, we define the annular type neighborhood

$$
\mathcal{A}(\mathcal{K}, r)=\left\{s e^{i \theta}: r^{-1} \leq s \leq r, \theta \in \mathcal{K}\right\}
$$

\section{Lemma 6.1}

Let $\Delta$ be a subinterval of $[-\pi, \pi)$ and $\Delta^{\prime}$ be a subinterval of $\Delta$ with both endpoints different from those of $\Delta$. Let $A>0$. There exists $n_{0}$ and $C$ such that for $n \geq n_{0}$ and polynomials $P$ of degree $\leq n$, we have

$$
\|P\|_{L_{\infty}\left(\mathcal{A}\left(\Delta^{\prime}, 1+\frac{A}{n}\right)\right)} \leq C\|P\|_{L_{\infty}(\mathcal{A}(\Delta, 1))} .
$$

Proof

After a rotation of the unit circle, we may assume that

$$
\Delta=[\alpha, 2 \pi-\alpha]
$$

and possibly by enlarging $\Delta^{\prime}$ after a similar rotation,

$$
\Delta^{\prime}=\left[\alpha^{\prime}, 2 \pi-\alpha^{\prime}\right],
$$


where $0 \leq \alpha<\alpha^{\prime}<\pi$. The conformal map of $\mathbb{C} \backslash \mathcal{A}(\Delta, 1)$ onto the exterior of the unit ball is [7], [12]

$$
\Psi(z)=\frac{1}{2 \cos \frac{\alpha}{2}}\left\{z+1+\sqrt{\left(z-e^{-i \alpha}\right)\left(z-e^{i \alpha}\right)}\right\} .
$$

The branch of the square root is taken so that it is analytic off $\mathcal{A}(\Delta, 1)$ and behaves like $z(1+o(1))$ as $z \rightarrow \infty$. If $P$ is a polynomial of degree $\leq n$, then $P / \Psi^{n}$ is analytic off $\mathcal{A}(\Delta, 1)$, and has a finite limit at $\infty$. By the maximum modulus principle, for all $z$ off $\Delta$,

$$
\left|P / \Psi^{n}\right|(z) \leq\|P\|_{L_{\infty}(\mathcal{A}(\Delta, 1))} .
$$

Of course, this is a special case of the Bernstein-Walsh inequality. It thus suffices to prove that

$$
|\Psi(r z)| \leq 1+\frac{C}{n}
$$

for $z \in \mathcal{A}\left(\Delta^{\prime}, 1\right)$ and $\left(1+\frac{A}{n}\right)^{-1} \leq r \leq 1+\frac{A}{n}$. For this we need only observe that

$$
\Psi^{\prime}(u)=\frac{1}{2 \cos \frac{\alpha}{2}}\left\{1+\frac{u-\cos \frac{\alpha}{2}}{\sqrt{\left(u-e^{-i \alpha}\right)\left(u-e^{i \alpha}\right)}}\right\}
$$

is bounded as long as $u$ stays away from the endpoints $e^{ \pm i \alpha}$ of $\mathcal{A}(\Delta, 1)$. Hence for some $C_{1}$ depending only on $A, \Delta$ and $\Delta^{\prime}$,

$$
|\Psi(r z)-\Psi(z)|=\left|\int_{z}^{r z} \Psi^{\prime}\right| \leq C_{1}|r-1| \leq C_{1} A / n .
$$

As $|\Psi(z)|=1$ for $z=e^{i \theta}, \theta \in \Delta^{\prime}$ (with a suitable interpretation of $\Psi(z)$ as a boundary value), we obtain (6.3).

\section{Lemma 6.2}

Assume the hypotheses of Theorem 1.1. Let $A>0$.

(a) There exists an open set $\mathcal{I}$, consisting of finitely many intervals, that contains $J$, and $n_{0}, C>0$ such that

$$
\frac{1}{n}\left|K_{n}(u, v)\right| \leq C
$$

for $n \geq n_{0}(A)$, and $u, v \in \mathcal{A}\left(\mathcal{I}, 1+\frac{A}{n}\right)$.

(b) Under the same restrictions as in (a), uniformly in $u, v \in \mathcal{A}\left(I, 1+\frac{A}{n}\right)$ and $a, b$ in a compact subset of the plane,

$$
\frac{1}{n}\left|K_{n}\left(u e^{i a / n}, v e^{i \bar{b} / n}\right)-K_{n}\left(u\left(1+\frac{i a}{n}\right), v\left(1+\frac{i \bar{b}}{n}\right)\right)\right|=o(1) .
$$

\section{Proof}

(a) As $J$ is compact, and $w$ is continuous and positive at each point of $J$, we can find an open set $\mathcal{J}$ containing $J$ and $C>0$ such that

$$
w \geq C \text { in } \mathcal{J} .
$$


We may assume that $\mathcal{J}$ consists of finitely many open intervals (again because of the compactness of $J$ ). If we take $\mu^{*}$ to be the measure equal to $\mu$ outside $\mathcal{J}$, and equal to $C d \theta$ in $\mathcal{J}$, we have $d \mu \geq d \mu^{*}$, so

$$
\Omega_{n} \geq \Omega_{n}^{*} \text { everywhere. }
$$

Moreover, Theorem 3.1 shows that $\Omega_{n}^{*} \sim \frac{1}{n}$ uniformly in compact subsets of $\mathcal{J}$. Note here that $\mu^{*}$ is regular, and this follows from Theorem 5.3.3 in [25, p. 148]. Then if we take $\mathcal{K}$ to consist of finitely many intervals, each smaller than, but concentric, with those in $\mathcal{J}$, but with different endpoints, we obtain

$$
\Omega_{n} \geq \frac{C}{n} \text { in } \mathcal{K} .
$$

That is,

$$
\frac{1}{n}\left|K_{n}\left(e^{i s}, e^{i s}\right)\right| \leq C, s \in \mathcal{K} .
$$

By Cauchy-Schwarz, we also obtain

$$
\frac{1}{n}\left|K_{n}\left(e^{i s}, e^{i t}\right)\right| \leq C, s, t \in \mathcal{K} .
$$

Now let $\mathcal{I}$ consist of finitely many intervals, each smaller than, but concentric, with those in $\mathcal{K}$, but with different endpoints. Applying Lemma 6.1 in each variable, and (6.6), to each of the subintervals of $\mathcal{I}$, gives (6.4).

(b) By Cauchy's estimates, if $g$ is a function analytic in the closed ball centre $a$, radius $\frac{1}{n}$, we have

$$
\left|g^{\prime}(a)\right| \leq n \max _{|t-a|=\frac{1}{n}}|g(t)| .
$$

Applying this in each variable to $\frac{1}{n} K_{n}(u, v)$ and using (a), we deduce that

$$
\left|\frac{\partial}{\partial u} K_{n}(u, v)\right|+\left|\frac{\partial}{\partial v}\left[K_{n}(u, \bar{v})\right]\right| \leq C n^{2}
$$

for $u, v \in \mathcal{A}\left(I, 1+\frac{A}{n}\right)$. (If necessary, we can slightly shrink $\mathcal{I}$. Recall too that $K_{n}(u, \bar{v})$ is analytic in $\left.u, v\right)$. Finally, we use

$$
\begin{aligned}
& K_{n}\left(u e^{i a / n}, v e^{i \bar{b} / n}\right)-K_{n}\left(u\left(1+\frac{i a}{n}\right), v\left(1+\frac{i \bar{b}}{n}\right)\right) \\
= & K_{n}\left(u e^{i a / n}, v e^{i \bar{b} / n}\right)-K_{n}\left(u\left(1+\frac{i a}{n}\right), v e^{i \bar{b} / n}\right) \\
& +K_{n}\left(u\left(1+\frac{i a}{n}\right), v e^{i \bar{b} / n}\right)-K_{n}\left(u\left(1+\frac{i a}{n}\right), v\left(1+\frac{i \bar{b}}{n}\right)\right) \\
= & \int_{u\left(1+\frac{i a}{n}\right)}^{u e^{i a / n}} \frac{\partial}{\partial t} K_{n}\left(t, v e^{i \bar{b} / n}\right) d t+\int_{v\left(1+\frac{i \bar{b}}{n}\right)}^{\frac{\partial}{\partial i \bar{b} / n}\left[\bar{s}\left[K_{n}\left(u\left(1+\frac{i a}{n}\right), s\right)\right]\right.} d s \\
= & O\left(n^{2}\right)\left|u e^{i a / n}-u\left(1+\frac{i a}{n}\right)\right|+O\left(n^{2}\right)\left|v e^{i \bar{b} / n}-v\left(1+\frac{i \bar{b}}{n}\right)\right|=O(1),
\end{aligned}
$$


so $(6.5)$ is true in the stronger from where $o(1)$ is replaced by $O\left(\frac{1}{n}\right)$.

\section{Theorem 6.3}

Assume the hypotheses of Theorem 1.1. Then

$$
\lim _{n \rightarrow \infty} \frac{K_{n}\left(z\left(1+\frac{i 2 \pi a}{n}\right), z\left(1+\frac{i 2 \pi \bar{b}}{n}\right)\right)}{K_{n}(z, z)}=e^{i \pi(a-b)} \frac{\sin \pi(a-b)}{\pi(a-b)},
$$

uniformly for $a, b$ in compact subsets of the plane and $z=e^{i \theta}, \theta \in J$.

Proof

It follows from the lemma above, that $\left\{\frac{1}{n} K_{n}\left(z\left(1+\frac{i 2 \pi a}{n}\right), z\left(1+\frac{i 2 \pi \bar{b}}{n}\right)\right)\right\}_{n}$ is uniformly bounded for $a, b$ in compact subsets of the plane and for $z=e^{i \theta}$, $\theta \in \mathcal{I}$. Moreover, it is a sequence of polynomials in $a, b$. It follows from Theorem 1.1 and Lemma 6.2(b), that uniformly for $a, b$ in compact subsets of the real line, we have the limit (6.7) above. Convergence continuation theorems give the limit uniformly for $a, b$ in compact subsets of the plane. However, the uniformity in $\theta$ has to be proved separately. For this, choose a sequence $\left\{\theta_{n}\right\}$ in $J$ and let $z_{n}=e^{i \theta_{n}}$ for $n \geq 1$. Theorems 1.1 and Lemma $6.2(\mathrm{~b})$ show that

$$
\lim _{n \rightarrow \infty} \frac{K_{n}\left(z_{n}\left(1+\frac{i 2 \pi a}{n}\right), z_{n}\left(1+\frac{i 2 \pi \bar{b}}{n}\right)\right)}{K_{n}\left(z_{n}, z_{n}\right)}=e^{i \pi(a-b)} \frac{\sin \pi(a-b)}{\pi(a-b)}
$$

uniformly for $a, b$ in compact subsets of the real line. Moreover, by Lemma $6.2(\mathrm{a}),\left\{\frac{K_{n}\left(z_{n}\left(1+\frac{i 2 \pi a}{n}\right), z_{n}\left(1+\frac{i 2 \pi \bar{b}}{n}\right)\right)}{K_{n}\left(z_{n}, z_{n}\right)}\right\}$ is uniformly bounded for $a, b$ in compact subsets of the plane. Then (6.8) holds uniformly for $a, b$ in compact subsets of the plane. This implies the stated uniformity in $z$.

\section{Proof of Corollary $\mathbf{1 . 2}$}

Exactly as in the proof of Theorem 2.2, we have the Taylor series identity (2.9) for $K_{n}$ instead of $K_{n}^{L}$. Moreover, from Theorem 6.3, we have the uniform convergence of $\frac{1}{K_{n}(z, z)} K_{n}\left(z\left(1+\frac{i 2 \pi a}{n}\right), z\left(1+\frac{i 2 \pi \bar{b}}{n}\right)\right)$ for $a, b$ in compact subsets of the complex plane. Recalling the identity (2.7) from Theorem 2.1, the result now follows, for each fixed $z=e^{i \theta}, \theta \in J$. However, the uniformity in $\theta$ still must be proved separately. For this, we just need to choose a sequence $\left\{\theta_{n}\right\}$ as in the proof of Theorem 6.3, and proceed as there.

\section{REFERENCES}

[1] J. Baik, T. Kriecherbauer, K. T-R. McLaughlin, P.D. Miller, Uniform Asymptotics for Polynomials Orthogonal with respect to a General Class of Discrete Weights and Universality Results for Associated Ensembles, Princeton Annals of Mathematics Studies, 2006. 
[2] T. Bloom, N. Levenberg, Strong Asymptotics of Christoffel functions for Planar Measures, manuscript.

[3] R. DeVore, G.G. Lorentz, Constructive Approximation, Springer, Berlin, 1993.

[4] P. Deift, Orthogonal Polynomials and Random Matrices: A Riemann-Hilbert Approach, Courant Institute Lecture Notes, Vol. 3, New York University Pres, New York, 1999.

[5] P. Deift, Integrable Operators, (in) Differential Operators and Spectral Theory, Translations of the American Mathematical Society Series 2, 189(1999), 69-84.

[6] P. Deift, T. Kriecherbauer, K. T-R. McLaughlin, S. Venakides and X. Zhou, Uniform Asymptotics for Polynomials Orthogonal with respect to Varying Exponential Weights and Applications to Universality Questions in Random Matrix Theory, Communications in Pure and Applied Maths., 52(1999), 1335-1425.

[7] L. Golinskii, Akhiezer's Orthogonal Polynomials and Bernstein-Szegö method for a circular arc, J. Approx. Theory, 95(1998), 229-263.

[8] A.B. Kuijlaars and M. Vanlessen, Universality for Eigenvalue Correlations from the Modified Jacobi Unitary Ensemble, International Maths. Research Notices, 30(2002), 1575-1600.

[9] Y. Last and B. Simon, Fine structure of the zeros of orthogonal polynomials, IV. A priori bounds and clock behavior, to appear in Comm. Pure Appl. Math.

[10] Eli Levin and D.S. Lubinsky, Universality Limits for Exponential Weights, manuscript.

[11] Eli Levin and D.S. Lubinsky, Applications of Universality Limits to Zeros and Reproducing Kernels of Orthogonal Polynomials, to appear in Journal of Approximation Theory.

[12] D.S. Lubinsky, $L_{p}$ Markov-Bernstein Inequalities on Arcs of the Circle, J. Approx. Theory, 108(2001), 1-17.

[13] D.S. Lubinsky, A New Approach to Universality Limits involving Orthogonal Polynomials, to appear in Annals of Mathematics.

[14] D.S. Lubinsky, A New Approach to Universality Limits at the Edge of the Spectrum, to appear in Contemporary Mathematics (Proceedings of the 60th birthday conference of P. Deift).

[15] A. Martinez-Finkelshtein, K. T.-R. McLaughlin, and E. B. Saff, Szegö orthogonal polynomials with respect to an analytic weight: canonical representation and strong asymptotics, Constructive Approximation 24(2006), 319-363.

[16] A. Mate, P. Nevai, V. Totik, Szego's Extremum Problem on the Unit Circle, Annals of Math., 134(1991), 433-453.

[17] K. T.-R. McLaughlin and P. D. Miller, The D Steepest Descent Method and the Asymptotic Behavior of Polynomials Orthogonal on the Unit Circle with Fixed and Exponentially Varying Nonanalytic Weights, International Mathematics Research Papers (2006), 1-78.

[18] M.L. Mehta, Random Matrices, 2nd edn., Academic Press, Boston, 1991.

[19] P. Nevai, Orthogonal Polynomials, Memoirs of the AMS no. 213 (1979).

[20] P. Nevai, Geza Freud, Orthogonal Polynomials and Christoffel Functions: A Case Study, J. Approx. Theory, 48(1986), 3-167.

[21] F. Riesz and B. Sz-Nagy, Functional Analysis, Dover, New York, 1990.

[22] B. Simon, Orthogonal Polynomials on the Unit Circle, Parts 1 and 2, American Mathematical Society, Providence, 2005.

[23] B. Simon, Fine structure of the zeros of orthogonal polynomials, I. A tale of two pictures, Electronic Transactions on Numerical Analysis 25 (2006), 328-268.

[24] B. Simon, Rank One Perturbations and Zeros of Paraorthogonal polynomials on the Unit Circle, to appear in J. Math. Anal. Appl.

[25] H. Stahl and V. Totik, General Orthogonal Polynomials, Cambridge University Press, Cambridge, 1992. 
[26] G. Szegő, Orthogonal Polynomials, 4th edn., American Mathematical Society Colloquium Publications, Vol. 23, American Math. Soc. Providence, 1975.

[27] V. Totik, Asymptotics for Christoffel Functions for General Measures on the Real Line, J. d'Analyse Math., 81(2000), 283-303.

[28] M.W. Wong, First and Second Kind Paraorthogonal Polynomials and Their Zeros, to appear in J. Approx. Theory.

${ }^{1}$ Mathematics Department, The Open University of Israel, P.O. Box 808, Raanana 43107, Israel., ${ }^{2}$ School of Mathematics, Georgia Institute of Technology, Atlanta, GA 30332-0160, USA., ${ }^{1}$ LUbinsky@Math.GateCh.Edu, ${ }^{2}$ EliLe@openu.AC.IL 